\title{
Article \\ Statistical Analysis and Neural Network in Detecting Steel Cord Failures in Conveyor Belts
}

\author{
Dominika Olchówka $^{1}{ }^{\mathbb{D}}$, Aleksandra Rzeszowska ${ }^{2, *}$, Leszek Jurdziak ${ }^{1}$ and Ryszard Błażej ${ }^{1}$ \\ 1 Faculty of Geoengineering Mining and Geology, Wroclaw University of Science and Technology, Na Grobli 15, \\ 50-421 Wroclaw, Poland; dominika.olchowka@pwr.edu.pl (D.O.); leszek.jurdziak@pwr.edu.pl (L.J.); \\ ryszard.blazej@pwr.edu.pl (R.B.) \\ 2 Faculty of Electronics, Wroclaw University of Science and Technology, Janiszewskiego 11/17, \\ 50-372 Wroclaw, Poland \\ * Correspondence: aleksandra.rzeszowska@pwr.edu.pl
}

check for updates

Citation: Olchówka, D.; Rzeszowska, A.; Jurdziak, L.; Błażej, R. Statistical Analysis and Neural Network in Detecting Steel Cord Failures in Conveyor Belts. Energies 2021, 14 , 3081. https://doi.org/10.3390/ en14113081

Academic Editors: Daniela Marasová, Monika Hardygora and

Mirosław Bajda

Received: 6 May 2021

Accepted: 24 May 2021

Published: 26 May 2021

Publisher's Note: MDPI stays neutral with regard to jurisdictional claims in published maps and institutional affiliations.

Copyright: (c) 2021 by the authors. Licensee MDPI, Basel, Switzerland. This article is an open access article distributed under the terms and conditions of the Creative Commons Attribution (CC BY) license (https:// creativecommons.org/licenses/by/ $4.0 /)$.

\begin{abstract}
This paper presents the identification and classification of steel cord failures in the conveyor belt core based on an analysis of a two-dimensional image of magnetic field changes recorded using the Diagbelt system around scanned failures in the test belt. The obtained set of identified changes in images, obtained for numerous parameters settings of the device, were the base for statistical analysis. This analysis makes it possible to determine the Pearson's linear correlation coefficient between the parameters being changed and the image of the failures. In the second stage of the research, artificial intelligence methods were applied to construct a multilayer neural network (MLP) and to teach it appropriate identification of damage. In both methods, the same data sets were used, which made it possible to compare methods.
\end{abstract}

Keywords: conveyor belts; magnetic method; diagnostics; NDT method; belt damage; statistical analysis; neural networks

\section{Introduction}

The non-destructive testing (NDT) of conveyor belts gives vast possibilities related to the optimization of conveyor belt maintenance costs, such as choosing the right moment for repair, replacement or recondition of the belt based on, among other factors, detected damage [1] or the rate of change of belt thickness [2]. Belt operating time depends on several factors that are presented in the literature [2]. Among other things, it is affected by the hardness, size and shape of transported materials, the specificity of transport point and the length and age of belt cord. Some of these factors damage the belt covers or belt core. Figure 1 shows the cross-section of the example conveyor belt with steel cords.

NDT research use offers, inter alia, analysis of the magnetic field changes generated by damaged or missing cords. Research using this method has been carried out since 1970 [3]. Since then, several researchers around the world have developed various systems to detect damage to steel cords in the belt core [4-6]. One of the systems in use for studies is a Diagbelt magnetic system, which enables researchers to obtain two-dimensional images suitable for further analysis [7-9]. The device detects magnetic field changes arising during the movement of cord failures beneath the measuring probe (installed across the width of the belt) which generate a discredited signal $(-1,0$ or 1$)$ corresponding to measured values of the magnetic field. The positive value of the signal measured by the device is represented in the figures presented in this paper in blue, and the signal of the negative magnetic field is represented in yellow. 


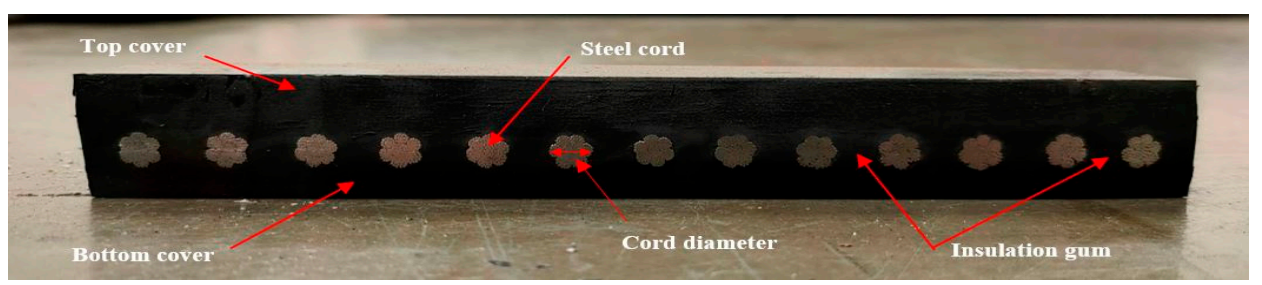

Figure 1. The cross-section of the belt with steel cords.

For the performed comparative analysis, a reference conveyor belt containing several artificial cord failures was used. The measurements were carried out by modifying system parameters: belt speed, the distance between the measuring probe and the cord and measurement sensitivity. These parameters were selected based on previous studies [10], which have confirmed their impact on received signals. The measurements were performed for many combinations of these parameters over ten measuring cycles, of which three were selected and used for the analysis. Preliminary visual evaluation of the data indicates the relationship between failure detection signal and the above parameters. Some of the damage with the appropriate settings of parameter values generated similar signals. Figure 2 gives an example of this situation (damage 3 and damage 1).

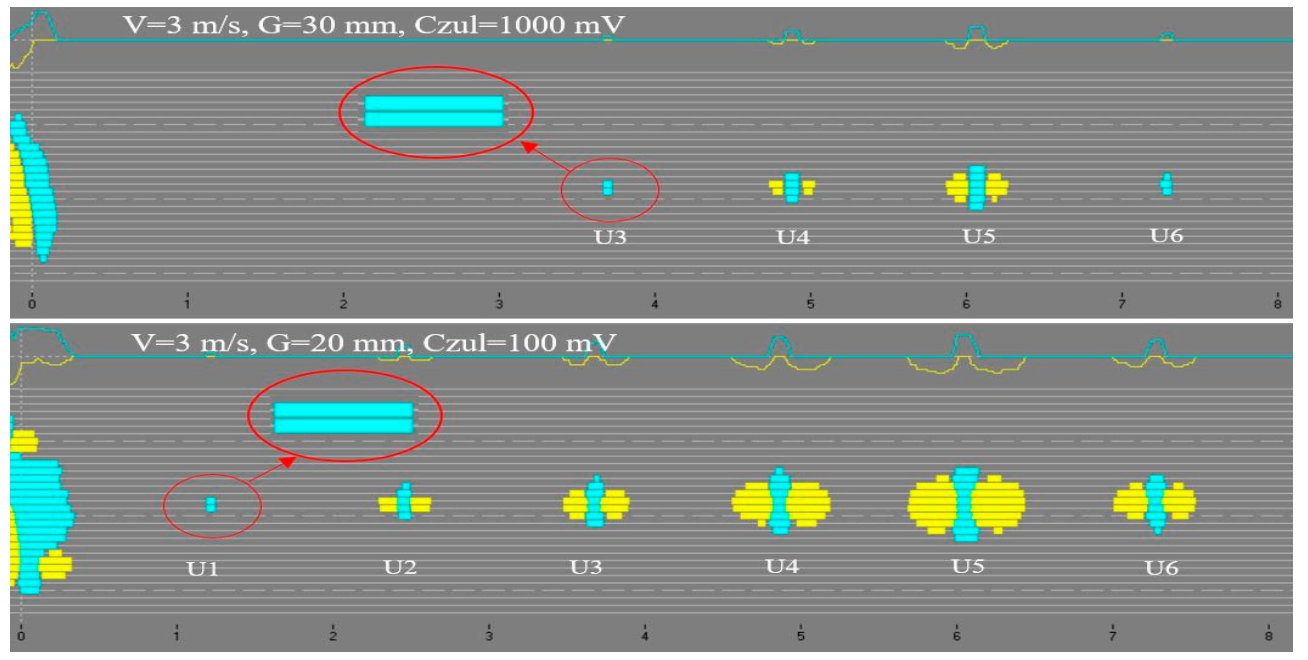

Figure 2. 2D images of the damage for significantly different settings of measuring equipment parameters.

The tested failures were divided into six categories: partial cord damage (20\% (U1) and $50 \%(\mathrm{U} 2)$ ) in one cord, complete cut of one steel cord (U3), cut of three (U4) and six (U5) cords and resection of one cord to the length of $20 \mathrm{~mm}$ (U6) (Figure 3).

\section{Preparation of Data for Analysis}

Each one of the cord failures described above generated a magnetic signal, and data were consolidated into 12 values, four for each of the sub-areas: magnetic signal surface areas, number of channels on which signal be detected, width and length of the signals. The method of calculating the size of signal for exemplary damage is shown in Figure 4 and described by Equations (1)-(3).

$$
\begin{gathered}
\text { Z1sum = Z1_1 + Z1_2 + Z1_3 + Z1_4 } \\
\text { Nsum = N1 + N2 + N3 + N4 + N5 + N6 } \\
\text { Z2sum = Z2_1 + Z2_2 + Z2_3 + Z2_4 }
\end{gathered}
$$


where: Z1_klength of the signal detected on the k-th channel for the signal before damage, Nklength of the signal detected on the k-th channel for the signal of damage and Z2_klength of the signal detected on the k-th channel for the signal behind damage.

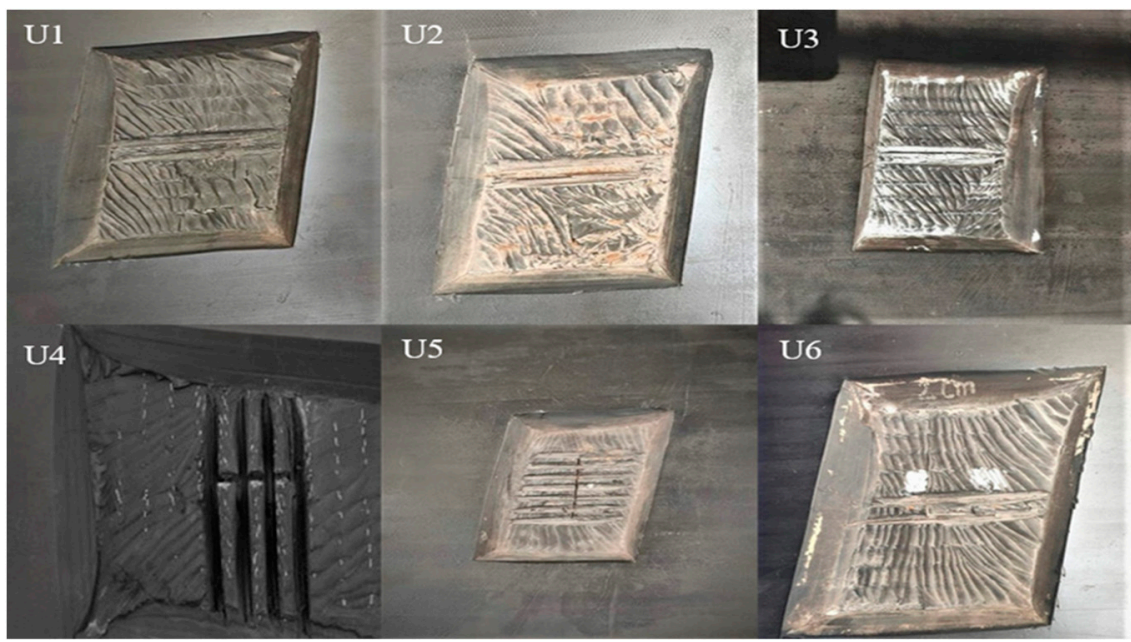

Figure 3. The actual appearance of the damage.

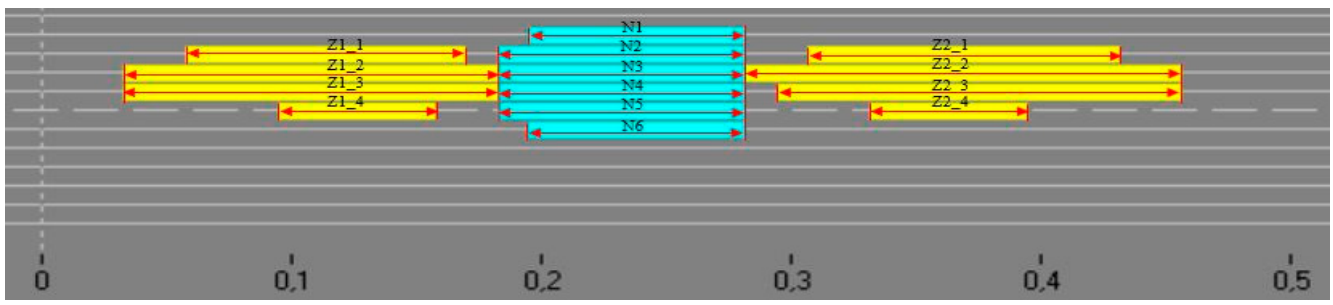

Figure 4. The method of calculating values describing selected damage.

During the measurements, the belt speed (V) was increased from 2 up to $5 \mathrm{~m} / \mathrm{s}$ (in increases of $1 \mathrm{~m} / \mathrm{s}$ ), the distance between the measuring probe and the cord ( $\mathrm{g}$ ) was changed within the range of 20-50 mm (in increases of $10 \mathrm{~mm}$ ) and the following sensitivity levels (c) were applied: $100 \mathrm{mV}, 150 \mathrm{mV}, 200 \mathrm{mV}, 250 \mathrm{mV}, 300 \mathrm{mV}, 400 \mathrm{mV}, 500 \mathrm{mV}, 600 \mathrm{mV}, 700 \mathrm{mV}$ and $1000 \mathrm{mV}$. The selection of these parameters was predicated on technical capabilities (speed of the test conveyor) and also the observation of system behaviour and its settings in numerous previous studies [10]. The above parameter values are shown on the axis in Figure 5 .

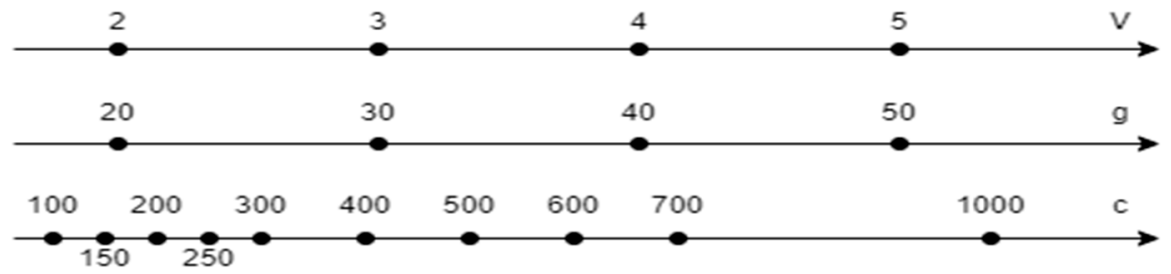

Figure 5. The distribution of measurement parameters.

The number of tested triple variants for given settings of the measuring system amounted to 160 .

$$
p=n_{c} \cdot n_{v} \cdot n_{g}=4 \cdot 10 \cdot 4=160
$$

where: $p$-quantity of triples parameters variants, $n_{c}$-number of settings of sensitivity parameter, $n_{v}$-number of settings of belt speed and $n_{g}$-number of settings of distance between the measuring probe and the cord. 
For each of the three measuring cycles for six defined types of damage, 2880 records describing the damage should be obtained.

$$
L_{p}=p \cdot l_{c} \cdot l_{k}=160 \cdot 3 \cdot 6=2880
$$

where: $L_{p}$-theoretical number of records, $l_{c}$-number of measuring cycles taken into account and $l_{k}-$ number of types of damage.

The actual amount of data was lower (2367), because magnetic field changes were not detected for less core damage in certain measurement settings.

One paper [11] defines the most appropriate measuring system parameters, which are presented in Table 1. For these ranges' apparatus settings (three parameters), the number of output data sets decreased to the value of:

$$
L_{p}=n_{c} \cdot n_{v} \cdot n_{g} \cdot l_{c} \cdot l_{k}=4 \cdot 1 \cdot(4+3+3+2) \cdot 3 \cdot 6=864
$$

Table 1. Preferred measurement system parameter settings.

\begin{tabular}{ccc}
\hline $\begin{array}{c}\text { Belt Speed } \\
{[\mathrm{m} / \mathbf{s}]}\end{array}$ & $\begin{array}{c}\text { Range between the Belt Core and the } \\
\text { Measuring Probe }[\mathrm{mm}]\end{array}$ & $\begin{array}{c}\text { Sensitivity } \\
{[\mathrm{mV}]}\end{array}$ \\
\hline 2 & $20-50$ & $200-300$ \\
3 & $20-50$ & $300-400$ \\
4 & $20-50$ & $400-500$ \\
5 & $20-50$ & $600-700$ \\
\hline
\end{tabular}

In reality, however, the number of records was 693 (some minor defects were not detected in specific measurement settings).

The actual sensitivities of the measuring device are inversely proportional to the parameter value called "sensitivity". When the value of this parameter is very low (e.g., $50-100 \mathrm{mV}$ ), the measuring system is extremely sensitive to the slightest field changes; however, the signal produced by the device is difficult to interpret. Images of the failures fuse and also appear to measure noise (Figure 6). Furthermore, when the value of this parameter is too large (i.e., when the system was set to be insensitive), minor damage may not have been registered, since it generates slight field changes which are outside the scope of sensitivity of the equipment.

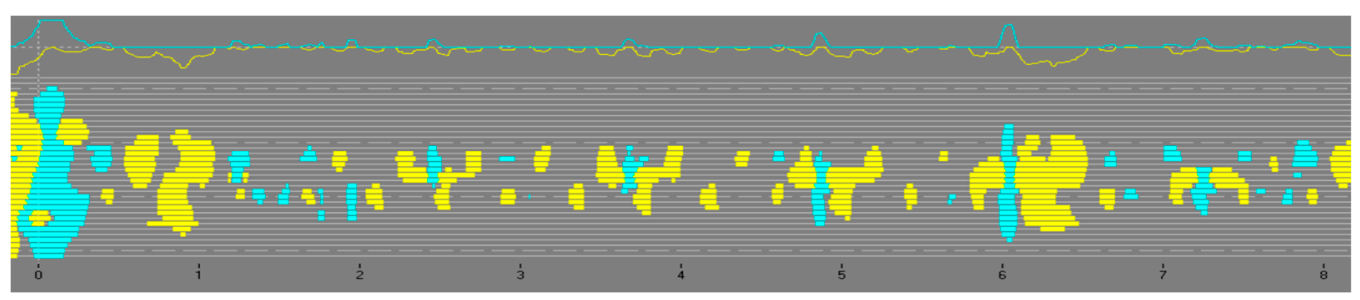

Figure 6. Inconclusive image of the damage for the measurement with high sensitivity of the measuring probe (scale $5 \mathrm{~mm} /$ pixel).

\section{Statistical Analysis}

The statistical analysis was performed only for the data obtained from the optimal sets of parameters (Table 2). The statistical analysis started with the verification of the obtained data to remove gross errors that may have appeared in the database, resulting, for example, from human oversight (e.g., entering incorrect data). In the next step, the correlation between the parameters taken into account in the analysis was examined. There are 13 such analysed values. They include damage number $\left(\mathrm{Nr}_{-} \mathrm{U}\right)$, number of cut cords (LL), area of damage (Pole_R), three parameters connected with the measurement system (belt speed-V, measuring probe distance- $\mathrm{G}$, sensitivity-Czul), the measurement cycle taken into account (Cycle) and the failure description, including two values for each of the 
three damage sub-areas (yellow field before damage $-\mathrm{Z} 1$, blue field $-\mathrm{N}$ and yellow field behind the damage Z2). The measured damage parameters are the sum of the lengths of the signals recorded in the measurement channels (Z1sum, Nsum, Z2sum) and the number of channels recording the signal related to a given sub-area (Z1_LK, N_LK, Z2_LK).

Table 2. Confidence intervals for the mean of the measured parameters.

\begin{tabular}{cccccccc}
\hline \multirow{2}{*}{ Z1sum } & & U1 & U2 & U3 & U4 & U5 & U6 \\
\multirow{2}{*}{ Z1_LK } & $\bar{x}$ & 0.00 & 3.09 & 45.72 & 373.48 & 793.65 & 130.91 \\
& $\Delta$ & 0.00 & 12.67 & 44.37 & 94.09 & 136.12 & 80.08 \\
\multirow{2}{*}{ Nsum } & $\bar{x}$ & 0.00 & 0.09 & 0.79 & 3.40 & 5.17 & 1.61 \\
& $\Delta$ & 0.00 & 0.08 & 0.23 & 0.17 & 0.21 & 0.27 \\
N_LK & $\bar{x}$ & 20.50 & 110.20 & 231.10 & 542.26 & 821.79 & 334.82 \\
& $\Delta$ & 2.07 & 8.29 & 13.12 & 16.09 & 25.25 & 14.44 \\
Z2sum & $\bar{x}$ & 0.88 & 2.29 & 3.68 & 6.01 & 8.04 & 4.46 \\
& $\Delta$ & 0.07 & 0.12 & 0.14 & 0.11 & 0.14 & 0.15 \\
\multirow{2}{*}{ Z2_LK } & $\bar{x}$ & 0.00 & 2.40 & 43.95 & 395.60 & 848.97 & 103.40 \\
& $\Delta$ & 0.00 & 3.83 & 14.06 & 32.92 & 45.95 & 21.77 \\
& $\bar{x}$ & 0.00 & 0.04 & 0.61 & 3.24 & 4.89 & 1.24 \\
& $\Delta$ & 0.00 & 0.05 & 0.18 & 0.19 & 0.18 & 0.23 \\
\hline
\end{tabular}

Figure 7 presents charts showing the values of six measured parameters for damage depending on the class to which the damage belongs. A visual evaluation of the data helps to decide whether a given parameter affects the class differentiation or is irrelevant and can be removed.
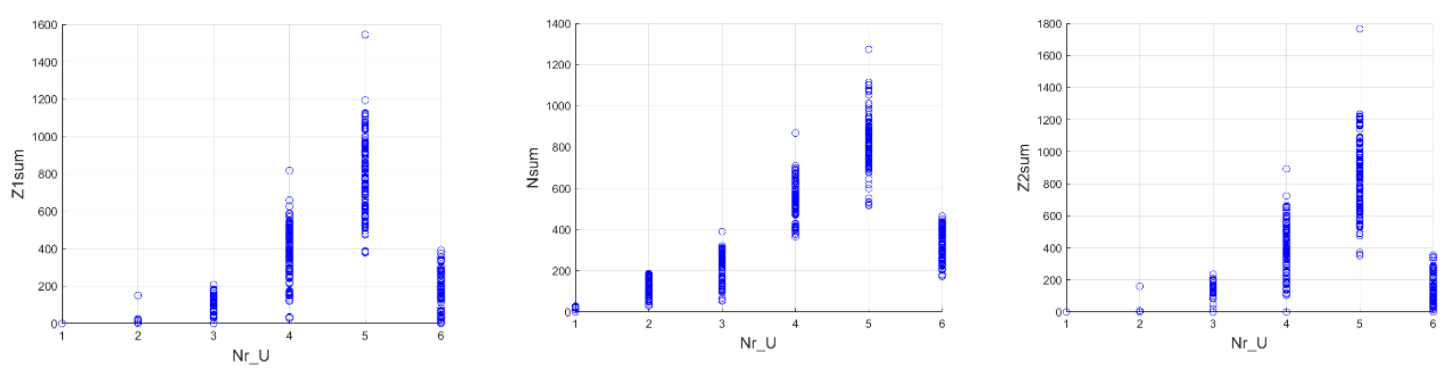

Figure 7. Distribution of the values of Z1sum, Nsum and Z2sum depending on the damage class.

The first part of the statistical analysis determined confidence intervals for the mean for each of the analysed measurements [12]. Confidence intervals for the mean are given as a formula:

$$
\bar{x} \pm \Delta=\bar{x} \pm 1.96 \frac{\sigma}{\sqrt{N}}
$$

where: $\bar{x}$-sample mean, $\Delta$ half the width of the confidence interval, $\sigma$ standard deviation and Nnumber of samples.

The input base is divided into two parts: a training set and a test set. Every third value went to the test set, while the remaining samples were left in the training set. The size of the training set was 462 samples, and the size of the test set was 231.

Table 2 summarizes the calculated values that facilitate the determination of the confidence interval for each of the analysed data sets.

For the data from the test, the set was determined and the mean value of each of the test sets was prepared in this way. These values were placed on the graph, which also marks the widths of the determined confidence intervals (Figure 8). Table 3 summarizes the results obtained from a given test group. In the table, the values that fall outside the designated confidence interval are marked in red. 


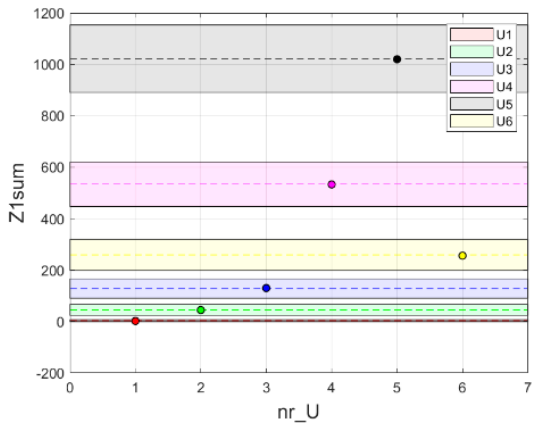

(a)

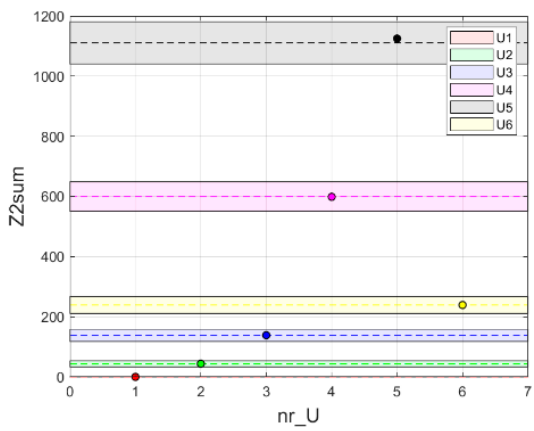

(c)

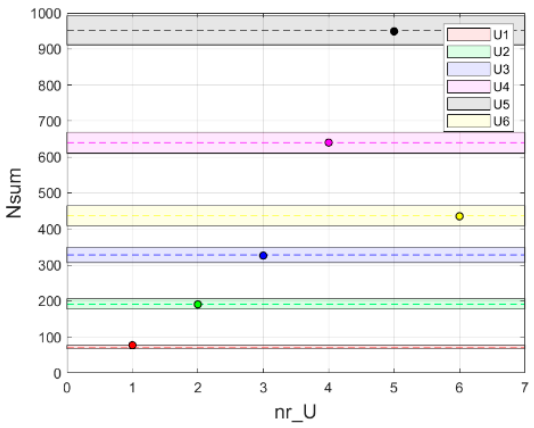

(b)

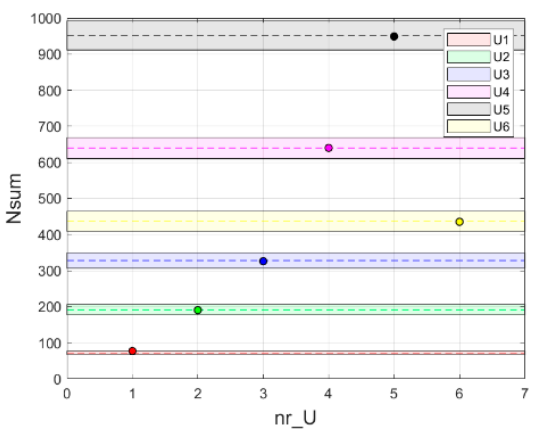

(d)

Figure 8. Confidence intervals test set, (a)-Z1sum (overlapping intervals), (b)-Nsum, (c)-Z2sum, (d)-N_LK (data outside of assigned value).

Table 3. The mean values of the test sets.

\begin{tabular}{lcccccc}
\hline & U1 & U2 & U3 & U4 & U5 & U6 \\
\hline Z1sum & 0.00 & 1.03 & 45.79 & 377.27 & 788.06 & 131.00 \\
Z1_LK & 0.00 & 0.06 & 0.75 & 3.40 & 5.17 & 1.63 \\
Nsum & 20.75 & 107.94 & 230.48 & 538.98 & 815.54 & 332.23 \\
N_LK & 1.00 & 2.26 & 3.67 & 6.04 & 8.04 & 4.46 \\
Z2sum & 0.00 & 0.00 & 42.33 & 400.04 & 843.58 & 104.23 \\
Z2_LK & 0.00 & 0.00 & 0.60 & 3.19 & 4.92 & 1.21 \\
\hline
\end{tabular}

Therefore, it can be noticed that the problem with recognition appears only in the case of data concerning the first type of damage. The number of channels in the test sample was mean 1.00, and in the training sample was $0.88 \pm 0.07$.

To check the influence of the analysed values on each other (their linear association), a statistical analysis was performed which determined the Pearson's product-moment linear correlation coefficients between the setting parameters of the measurement device and the sizes of output signals for each of the analysed failures. Pearson's correlation coefficient is a measure of linear correlation between two sets of data (the covariance of the two variables divided by the product of their standard deviations). Figure 9 displays the estimated correlations in the form of a matrix with coloured cells. Small changes to controlled parameters and lack of outliers in results allowed us to assume the linearity of changes in results. It was applied as an initial test of linear associations for further investigations to find the physical influence of settings changes on results, to select the best settings for given working conditions of conveyor belts and to select appropriate output parameters and methods for steel cord belt failures classification. 


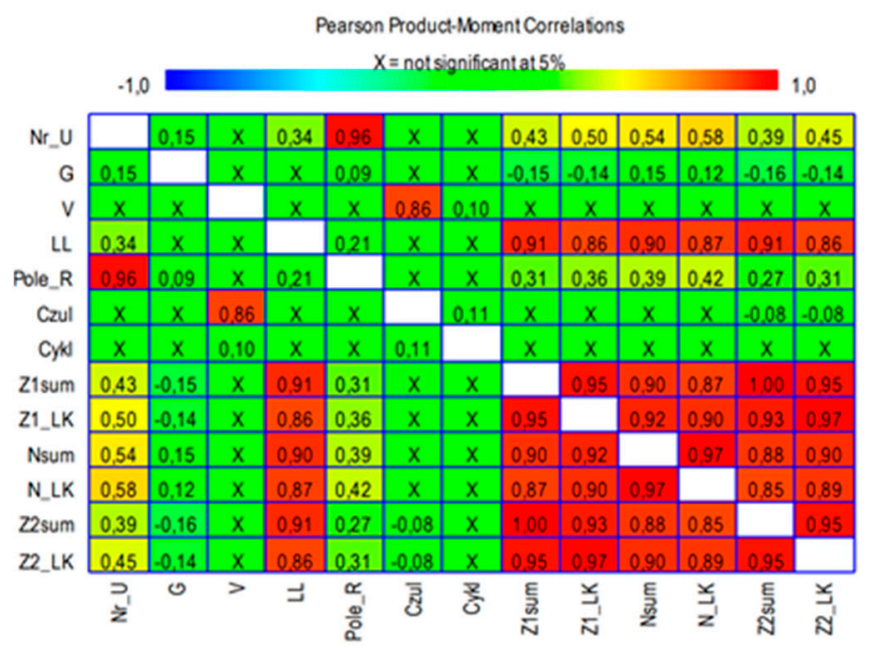

Figure 9. Pearson's linear correlation coefficients.

The data in the table above (Figure 9) are displayed using both colours and numerical values. Data marked with " $X$ " are statistically insignificant. Coefficients define a linear relationship between two different variables. The greater the value of the correlation parameter, the greater the degree of interconnection between the pairs of variables. It is worth noting that the presented table shows that the selected parameters of the measuring system did not significantly affect the type of damage. No correlation was found between the belt speed and the measurement results (statistically insignificant correlation), there is a low correlation between the measuring head distance and the measurement results (negative or positive, depending on the area, within the range $-0.16-0.15$ and no significant correlation or weak negative correlation $(-0.08)$ of the measurement results to the sensitivity of the device.

It is also worth noting that all measurement results are strongly positively correlated with each other, and the correlation between the values describing the yellow fields (Z1sum and Z2sum and Z1_LK and Z2_LK) is 1.00 and 0.97, which maintains the hypothesis about their symmetry [1].

Analogical statistical analysis for the full data set was described in detail in [13], but the results obtained there turned out to be less satisfactory than the results obtained for specific parameters of the measurement system. The distribution of the data from the complete set is shown in Figure 10 (these graphs show the values of Z1sum, Nsum and N_LK). These results largely overlap, and it is impossible to clearly define the boundaries of clusters [14,15].
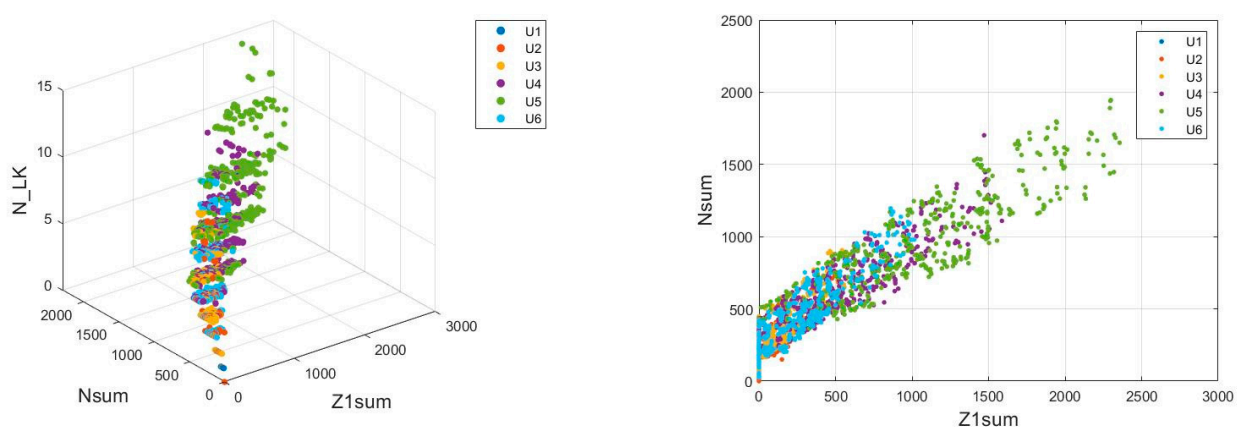

Figure 10. Cluster analysis—full data set, dependence Z1sum, Nsum, N_LK.

Similar graphs (Figure 11) were also plotted for the set of parameters of the measurement system tested in this analysis. It can be noticed that, in this case, it is possible to limit the obtained data with a certain curve marking the boundary of a given cluster, 
although there are still areas where the belonging of the measurement result to a given cluster is ambiguous.
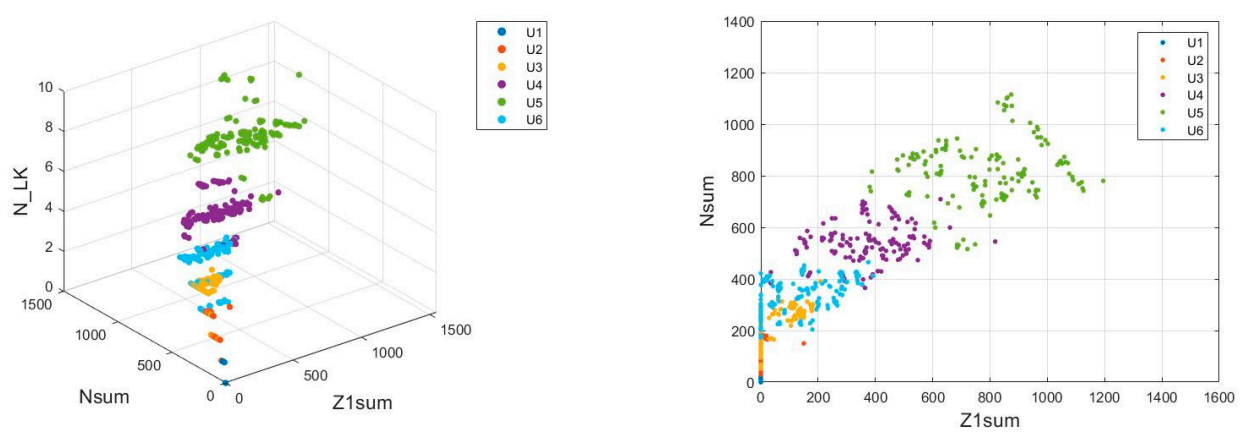

Figure 11. Cluster analysis—limited data set, dependence Z1sum, Nsum, N_LK.

Optical analysis of the obtained charts shows that the measurement data are highly probable to correctly classify the type of damage, but there are areas where the classification may fail because clusters overlap [15]. Due to this fact, another analysis was carried out using artificial neural networks.

\section{Analysis with the Use of Neural Networks}

The analysis of the selection of the structure and parameters of the neural network as well as the idea of its operation has been widely described in the literature. The studies [16-18] describe in detail the rationale behind the selection of specific parameters used in this research. The MATLAB software with the Deep Learning Toolbox installed was used for the learning process of neural networks. The way of using this toolbox is described in studies $[19,20]$. This toolbox enables the creation of a multilayer neural network with a given number of neurons in each of the hidden layers, and the ability to train the neural network on a given training data set (which is automatically divided into a training and validation set). Each layer of the artificial neural network is made up of individual computing units called neurons. Neurons, stimulated by the signal fed to their input, work out the output signal using the assigned weight $(w)$ and the added bias $(b)$. The process of training a neural network under supervision consists of repeatedly assessing it in the process of training samples from the test set, and then updating the weight based on the error between the network response and the expected response. To use artificial neural networks to classify the conveyor belt damage, it was necessary to generate appropriate sets of training and test sets, train the network on the training set, and then test it on the test set. Since neural networks can divide the classification of space non-linearly, over the course of this research two variants of the selection and division of the input data were distinguished. In each case, the vector of input data consisted of 15 elements (and this is the number of neurons in the input layer of the neural network): 3 measurement parameters and 4 values describing each of the three sub-areas. There are 6 neurons in the output layer-one each responsible for belonging to a given class of damage. Two hidden layers consisting of 31 and 63 neurons were placed between the input and output layers. The size of these layers was determined by the Kolmogorov theorem, according to which the number of neurons in the hidden layer should be equal to the number of neurons in the previous layer multiplied by two and increased by one [21]. The diagram of the neural network used in this study is shown in Figure 12. 


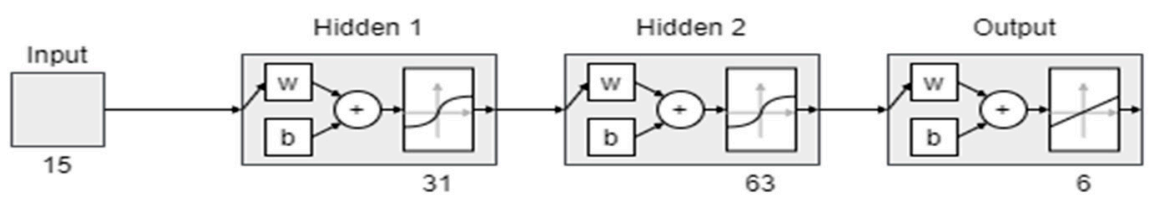

Figure 12. Diagram of the neural network used in the research.

Two variants were used for the process of learning neural networks:

- $\quad$ variant 1: analysis of all available data, two measurement cycles of each parameter set go to the training set, one cycle to the test set. The number of training and test sets was 1578 and 789.

- $\quad$ variant 2: analysis of data obtained in the measurements with the best possible sets of parameters (Table 1), two measurement cycles of each of the included parameter sets go to the training set, one cycle to the test set. The number of training and test sets was 462 and 231.

Each of the network learning processes in a given variant was performed three times, and the results are presented in Table 4 . The effectiveness of the diagnosis was divided depending on the type of damage that the neural network was supposed to recognize. In addition, the effectiveness of the diagnosis was also determined for the entire test set.

Table 4. Measurement results of neural networks analysis.

\begin{tabular}{ccccccccc}
\hline \multirow{2}{*}{ Variant } & No & \multicolumn{7}{c}{ Recognition Effectiveness [\%] } \\
\cline { 2 - 9 } & & U1 & U2 & U3 & U4 & U5 & U6 & Total \\
\hline \multirow{3}{*}{1} & 1 & 98.57 & 99.19 & 99.67 & 99.69 & 100.00 & 100.00 & 99.37 \\
& 2 & 97.14 & 97.56 & 98.68 & 98.13 & 100.00 & 100.00 & 98.86 \\
& 3 & 100.00 & 98.37 & 98.03 & 98.13 & 100.00 & 100.00 & 98.99 \\
\hline \multirow{2}{*}{2} & 1 & 100.00 & 97.14 & 97.92 & 100.00 & 100.00 & 100.00 & 99.13 \\
& 2 & 100.00 & 97.14 & 97.92 & 100.00 & 100.00 & 100.00 & 99.13 \\
& 3 & 100.00 & 100.00 & 100.00 & 100.00 & 100.00 & 100.00 & 100.00 \\
\hline
\end{tabular}

\section{Conclusions}

Many scans performed over the years using the Diagbelt system have shown that the magnetic measuring system is well suited to obtaining detailed information about the technical condition of the belt core. The idea of the system is based on the measurement of magnetic field changes at sites of core damage. The data obtained are presented on twodimensional pictures that can be easily analysed using proposed known methodologies. The results presented in this study confirm this observation, showing the high efficiency of identification of the type of damage using the signals generated by Diagbelt.

One such method is verification based on the average of the samples. For this purpose, from the set of training data the mean value and $95 \%$ confidence interval were calculated for each damage. Then, for the test set containing the specific damage data obtained at different set values (not taken for training), the mean value was determined, which allowed for its comparison with the previously determined confidence intervals. In nearly every instance, testing set data have been included in the relevant confidence interval. The number of samples in training and testing data for selected parameter sets was 8 and 4 , respectively, which could be a too-small value. It is, however, worth noting that with such a choice of analysis for automatic recognition of damage, it is necessary to execute many damage measurements for multiple sets of parameters to obtain a sample that comes from the same distribution as the training data. This solution can be cumbersome and, as the study shows, verification by the mean of the sample data is not always reliable.

An analysis of the Pearson correlation coefficient allows an assessment of the interdependence of evaluated parameters and therefore initially verifies which parameters are worth analysing with the classification of measuring damage, and which are redundant 
and have no correlation with the type of damage. Such an analysis does not allow for the designated similar data based on a new sample, but on its basis it is possible to construct a statistical model necessary for the assessment of future data. Creating such a model, which is a response to the analysis presented in this article, is a good direction for future research.

When the full set of data is limited to data obtained for the most appropriate system settings, better statistical analysis values are achieved. The full data set shown in the twodimensional and three-dimensional plots (Figures 9 and 10) indicates that these data cannot be isolated from each other and locked in separate clusters; however, for limited data, cluster analysis is possible, as areas of interdependent neighbouring clusters are slight.

The analysis based on neural networks allows the omission of the problem of nonlinearity. The network containing two hidden layers allows investigators to solve almost every problem of classification, provided it has the appropriate input data. In the framework of this explanation, analysis was carried out using neural networks, both on the complete dataset and the limited dataset containing results obtained from best possible system settings. The data collected in both of these variants have shown good efficacy (above 98\%) following the implementation of the testing process.

The network does not have a problem with classification of the last two types of damage (U5 and U6); however, it makes errors recognising defects $2-4$. This may be due to the real size of the defect concerned. The neural network analysis, compared to the statistical analysis, allows for quick action of the entire system while maintaining high efficiency.

It is worth noting that while analysis of statistical methods has already been used in the classification of belts damage, cluster analysis and analysis using the neural networks have so far been rarely discussed and their results rarely presented. Developers of the diagnostic systems, in many cases, prefer to retain the ability to interpret measurement results for themselves, so that their services will not become unnecessary. In the Industry 4.0 era [22], the automatic interpretation of the diagnostic signal is necessary to cope with data processing for an ever-increasing amount of data. Test results discussed in this paper are promising, and they show the direction of further action that authors are taking as part of the research project "Integrated mobile system of automatic testing and continuous diagnostics of the condition of conveyor belts" (project number: POIR.01.01.0100-1194/19) [23].

Author Contributions: Conceptualization, R.B. and L.J.; methodology, D.O., A.R. and L.J.; formal analysis, A.R. and L.J.; resources, R.B.; data curation, D.O., A.R.; writing-original draft preparation, D.O. and A.R.; writing-review and editing, R.B and L.J.; supervision, R.B. All authors have read and agreed to the published version of the manuscript.

Funding: This research received no external funding.

Institutional Review Board Statement: Not applicable.

Informed Consent Statement: Not applicable.

Data Availability Statement: The data presented in this study are available on request from the corresponding author.

Conflicts of Interest: The authors declare no conflict of interest.

\section{References}

1. Jurdziak, L.; Błażej, R.; Burduk, A.; Bajda, M.; Kirjanów-Błażej, A.; Kozłowski, T.; Olchówka, D. Optimization of the operating costs of conveyor beltsin a mine in various strategies of their replacement and diagnostics. Transp. Przemystowy Masz. Rob. 2020, 4, 14-22.

2. Webb, C.; Sikorska, J.; Khan, R.N.; Hodkiewicz, M. Developing and evaluating predictive conveyor belt wear models. Data-Cent. Eng. 2020, 1, e3. [CrossRef]

3. Barry, B. Multi-Channel Conveyor Belt Condition Monitoring. U.S. Patent 7,275,637, 2 October 2007.

4. Błażej, R. Assessment of the Technical Condition of Conveyor Belts with Steel Cords; Oficyna Wydawnicza Politechniki Wrocławskiej: Wrocław, Poland, 2018. (In Polish) 
5. Kwaśniewski, J. Magnetic Testing of Steel Ropes_Personnel Certification System in the MTR Method; Wydawnictwa AGH: Kraków, Poland, 2010. (In Polish)

6. Kwaśniewski, J.; Machula, T. Diagnostics of belts with steel cords. Transp. Przemysłowy 2006, 4, 22-24. (In Polish)

7. Błażej, R.; Jurdziak, L.; Kirjanów, A.; Kozłowski, T. Evaluation of the quality of steel cord belt splices based on belt condition examination using magnetic techniques. Diagnostyka 2015, 16, 59-64.

8. Błażej, R.; Jurdziak, L.; Kozłowski, T.; Kirjanów, A. The use of magnetic sensors in monitoring the condition of the core in steel cord conveyor belts-Tests of the measuring probe and the design of the DiagBelt system. Measurement 2018, 123, 48-53. [CrossRef]

9. Kirjanów, A.; Burduk, R. Regression method-based analysis of damage development in the core of steel cord conveyor belts. In Proceedings of the XVIII Conference of PhD Students and Young Scientists, Szklarska Poręba, Poland, 22-25 May 2018 ; p. 5.

10. Kozłowski, T.; Błażej, R. 2018 Influence of selected parameters on the magnetic testing results in a DiagBelt system. Transp. Przemystowy Masz. Rob. 2018, 4, 6-9. (In Polish)

11. Olchówka, D. Selection of Measurement Parameters Using the DiagBelt Magnetic System on the Test Conveyor. Master's Thesis, Wroclaw University of Science and Technology, Wroclaw, Poland, 2020. (In Polish)

12. Hazra, A. Using the confidence interval confidently. J. Thorac. Dis. 2017, 9, 4125-4130. [CrossRef] [PubMed]

13. Olchówka, D.; Jurdziak, L.; Błażej, R.; Kozłowski, T. Examination of the steel cord belts core damage condition using the DiagBelt system Part 1. Determining the number of steel cord cuts in damage. Transp. Przemystowy Masz. Rob. 2021, 1, 2-10. (In Polish)

14. Omran, M.; Engelbrecht, A.; Salman, A. An overview of clustering methods. Intell. Data Anal. 2007, 11, 583-605. [CrossRef]

15. Saxena, A.; Prasad, M.; Gupta, A.; Bharill, N.; Patel op Tiwari, A.; Er, M.; Lin, C. A Review of Clustering Techniques and Developments. Neurocomputing 2017, 267, 664-681. [CrossRef]

16. Hornik, K.; Stinchcombe, M.; White, H. Multilayer feedforward networks are universal approximators. Neural Netw. 1989, 2, 359-366. [CrossRef]

17. Osowski, S. Neural Networks for Information Processing; Oficyna Wydawnicza Politechniki Warszawskiej: Warszawa, Poland, 2020. (In Polish)

18. Phil, K. MATLAB Deep Learning: With Machine Learning, Neural Networks and Artificial Intelligence; Apress: Berkeley, CA, USA, 2017.

19. Anghel, D.; Ene, A.; Belu, N. A Matlab Neural Network Application for the Study of Working Conditions. Adv. Mater. Res. 2013, 837, 310-315. [CrossRef]

20. Demuth, H.; Beale, M. Neural Network Toolbox User's Guide; MathWorks, Inc.: Portola Valley, CA, USA, 2014.

21. Osowski, S. Neural Networks in Algorithmic Use; Wydawnictwo Naukowo Techniczne: Warszawa, Poland, 1996. (In Polish)

22. Jurdziak, L.; Błażej, R.; Bajda, M. Conveyor belt 4.0, Intelligent systems in production engineering and maintenance. Adv. Intell. Syst. Comput. 2019, 835, 645-654.

23. Integrated Mobile System of Automatic Testing and Continuous Diagnostics of the Condition of Conveyor Belts. Available online: https:/ / mapadotacji.gov.pl/projekty/1179135/ (accessed on 30 April 2021). 